\title{
XWebProcess: Agile Software Development for Web Applications
}

\author{
Américo Sampaio ${ }^{1}$, Alexandre Vasconcelos ${ }^{1}$, and Pedro R. Falcone Sampaio ${ }^{2}$ \\ ${ }^{1}$ Centro de informática, Universidade Federal de Pernambuco \\ Recife, Pernambuco - Brazil \\ $\{$ atfs, amlv\}@cin.ufpe.br \\ ${ }^{2}$ Computation Department, University of Manchester Institute of Science and Technology \\ United Kingdom \\ \{p.sampaio\}@co.umist.ac.uk
}

\begin{abstract}
Accelerating application development and reducing time to market is a highly valuable feature of a software process. For web-based applications, in particular, project development efforts often need to comply with severe time constraints imposed by the strategic business importance of the web. In this paper we describe XWebProcess, an agile process for web-based application development. XWebProcess is grounded on the principles underlying Extreme Programming and is aimed at building high quality web applications in a time effective way. XWebProcess' agility has been compared to XP via an experimental setting which shows its superiority in supporting web development dimensions such as requirements gathering, user navigation design, and software testing, while retaining the agile property of Extreme Programming.
\end{abstract}

\section{XWebProcess}

Some core elements of web application engineering that XWebProcess addresses are:

- Non-functional requirements such as concurrency, load balancing, security and distribution, which play an important role in the operation of web applications. The number of concurrent users can be substantial and appropriate software and hardware infrastructure may also be necessary.

- Different kinds of clients accessing the system via distinct browsers and protocols. Therefore it is important to identify different OS and hardware architectures underlying the system.

- Navigation and presentation aspects. In web applications, user interfaces often contain graphics, animations, links and text requiring attractive UI designs and simple navigability.

XWebProcess seeks to combine the key elements of Extreme Programming with process structures tailored to tackle the characteristics and factors of web engineering identified above, therefore providing an efficient and effective approach to the construction of web applications. 
The main reasons for leveraging Extreme Programming as the base software process are:

- XP is an agile process that is showing positive results in many software development projects. A recent survey presents some important quantitative data about a large number of XP projects built by different software companies worldwide. The projects varied in size, kind of application and application domain. Almost all XP projects finished on time and on budget and, among them, 28\% represented web projects.

- Recent contributions acknowledge the value of XP for web application development while recognizing the need for adaptations in the XP process to better suit the web application problem landscape.

- The wide availability of literature and project case studies simplifying the task of best practice identification.

The creation of XWebProcess can be summarized in four key steps. First, the must have features of a web engineering process were identified (e.g. disciplines, activities, artifacts, roles, etc.). Second, XP was modeled using OMG's software process engineering metamodel (SPEM) to obtain a better abstraction for representing the process and also to help its adaptation towards web application development. Third, adaptations of XP's elements and the inclusion of novel elements (disciplines) were performed to tailor the method to a web engineering framework. Finally, the process elements of XWebProcess were detailed and specified using SPEM.

$\mathrm{XWebProcess}$ is described in two views using SPEM to facilitate process construction. The use of SPEM enables an abstract description of the software process core elements (artifacts, roles, activities, etc.) and the description of how they relate to each other. The SPEM notation also helps to illustrate the adaptations and tailorings performed over XP to target web application development. SPEM was chosen due to its OMG standard status for software process modeling and due to the extensive endorsement provided by software companies such as IBM, Rational Software and Unisys.

The first view of the process model uses UML's activity diagram with the discipline stereotype defined in SPEM as shown in. This model helps to understand how the process behaves through time (dynamic view). The second view of the process details each discipline of the process using UML's class diagram alongside with SPEM stereotypes to represent common software process elements such as roles, activities and artifacts and the relationship among them.

It is important to mention that XWebProcess is a general web development process that does not depend on any specific technology, method, tool or technique. For instance, the process can be supported either with .NET or J2EE platforms. It also works with OOHDM or OOWS that are design methods for web applications. What is important to consider is if the specific method, tool or technology will have a negative impact on the agility of the process. 